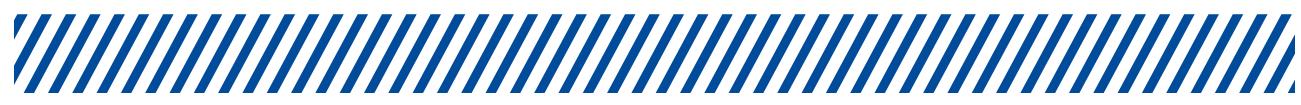

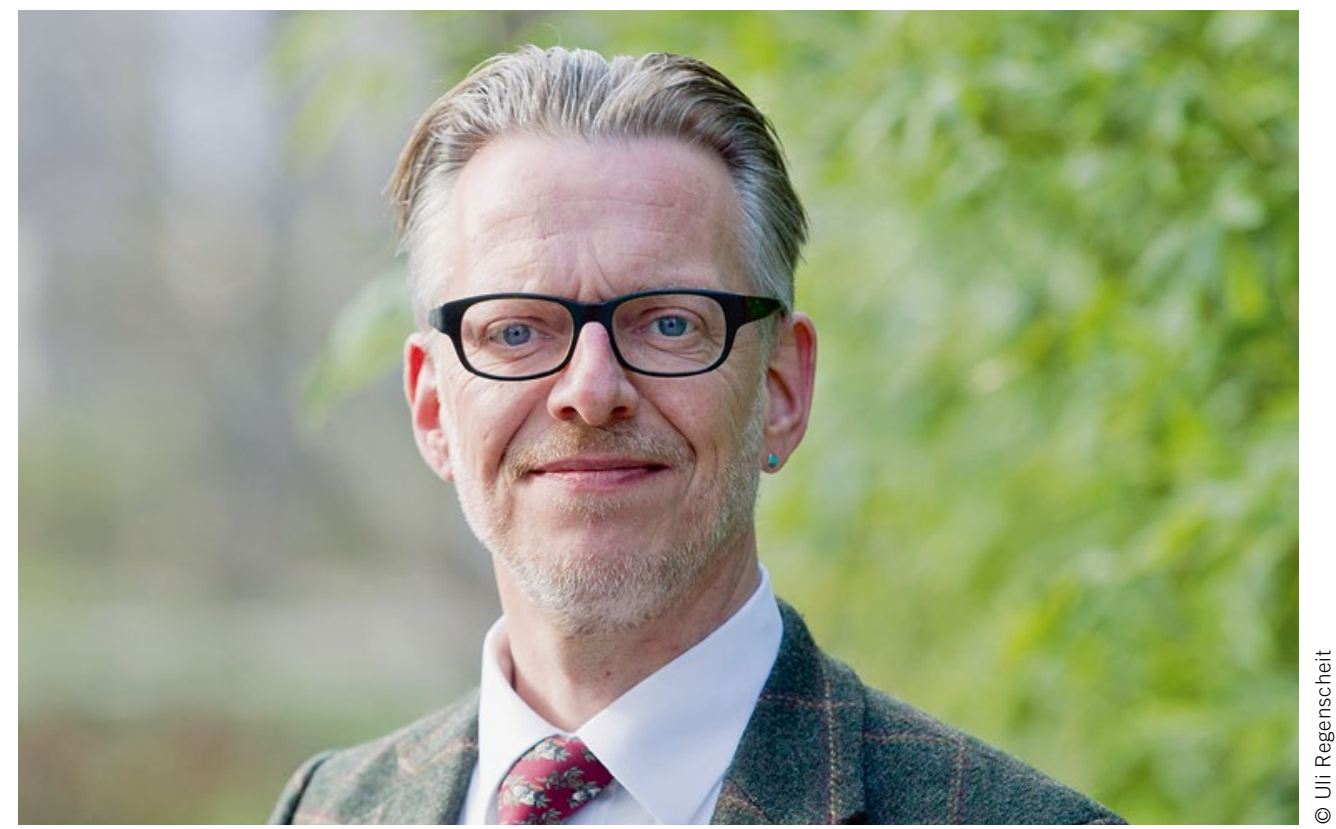

Dipl.-Ing. Torsten Buddenberg Head of Product Development, Mitsubishi Hitachi Power Systems Europe GmbH

\section{Künftige Mobilität ohne schlechtes Gewissen?}

Sind wir künftig alle in automatisch fahrenden Elektromobilen unterwegs, oder steuern wir auch im Jahr 2050 noch selber unsere großmotorigen SUVs? Das ist eine Frage, die nicht allein durch die Vernunft entschieden werden wird. Ein Auto ist kein reiner Kostenfaktor, sondern es ist ein wesentliches Zeichen für unsere Freiheit und gelebte Individualität. Kein Wunder, dass diese Diskussion oft emotional statt rational geführt wird.

Aber ist diese Emotionalität angemessen? Meiner Meinung nach: nein. Natürlich ändert sich die Art und Weise unserer Mobilität nicht über Nacht. Aber das ist in unserer Stromversorgung, der Branche, in der ich tätig bin, auch nicht passiert. Jede dritte in Deutschland verbrauchte Kilowattstunde ist heute regenerativ erzeugt. Auch die konventionelle Stromerzeugung ist effizienter und damit emissionsärmer geworden. Diese Entwicklung hat rund 15 Jahre gedauert (seit Inkrafttreten des ErneuerbareEnergien-Gesetzes) und sollte auf die Mobilität übertragbar sein.

Dafür braucht es viele Akteure, nicht nur aus der Automobilwirtschaft, sondern auch aus anderen Branchen wie dem Energieanlagenbau. So hat zum Beispiel Mitsubishi Hitachi Power Systems Europe gemeinsam mit Partnern bereits vor Jahren begonnen, neue Wege der Kraftstofferzeugung für die Mobilität zu untersuchen. Das Ergebnis: Wir können heute Großanlagen liefern, die Benzin aus regenerativem Strom erzeugen - und dabei den $\mathrm{CO}_{2}$-Anteil zwischen 70 bis $90 \%$ reduzieren.

Natürlich ist dieser Treibstoff teurer als das heute in Rotterdam gehandelte Benzin. Aber das ist zu kurz gedacht. In Deutschland muss heute die Petrochemie sicherstellen, dass der hier verbrauchte Treibstoff um 3,5 \% in seinem $\mathrm{CO}_{2}$-Ausstoß reduziert ist. Ansonsten ist, nach BImSchG §37, eine Strafzahlung von 470 Euro pro Tonne nicht erreichter $\mathrm{CO}_{2}$-Reduktion fällig. Wenn die Treibstoffversorger versäumen, diese Bedingungen zu erfüllen, werden die Strafen/ Kosten über den Treibstoffpreis auf alle Verbraucher umgelegt. Die zuvor genannte $\mathrm{CO}_{2}$-arme Benzinproduktion ist daher schon heute an vielen Standorten kostengünstiger möglich und die gesetzliche Reduktionsquote steigt.

Um solche Projekte umzusetzen, ist - neben dem Anlagenbau die Kooperation von bisher nebeneinander arbeitenden Branchen zwingend notwendig, ohne dass weitere Akteure dies boykottieren. Gemeint sind Energieversorgungsunternehmen, die Petrochemie und die Automobilindustrie. Eine nicht ganz einfache Aufgabe, da sich diese Branchen historisch weder in Bezug auf Innovationsfreude, Renditeerwartung und andere Kriterien vergleichen lassen. Für solche Kooperationen muss die Politik den Rahmen setzen. Das ist - neben den technischen Neuheiten - nicht nur eine Herausforderung, sondern auch eine Chance für Deutschland, das in diesem Bereich ein Zeichen setzen und für ganz Europa den Weg in eine $\mathrm{CO}_{2}$-ärmere Mobilität weisen kann.

Elektromobilität und/oder neue Verfahren zur Treibstoffherstellung sind nur zwei der möglichen Technologien, die uns zu diesem Ziel führen können. Aber um es zu erreichen, müssen Wirtschaft und Politik jetzt handeln und die richtigen Rahmenbedingungen setzen. Ansonsten werden Unternehmen und Politiker von der Entwicklung überrollt und müssen sehr wahrscheinlich mit kostspieligen Konsequenzen leben. Auch das haben wir alle - Verbraucher, Unternehmer, Politiker - aus den Umbrüchen bei der Stromversorgung schmerzhaft gelernt. 


\section{chassis tech}

7. Internationales Münchner Fahrwerk-Symposium

14. und 15. Juni 2016 I München

MODERNE FAHRWERKE

Etappen zu intelligenten Systemen

\section{SMART CHASSIS}

Anforderungen und Testing

\section{ASSISTENZSYSTEME}

Mehr Sicherheit und Komfort

I// WISSENSCHAFTLICHE LEITUNG

Prof. Dr. Peter E. Pfeffer

Hochschule München

I// EINE FÜR ALLE

\section{Vier Kongresse}

in einer Veranstaltung

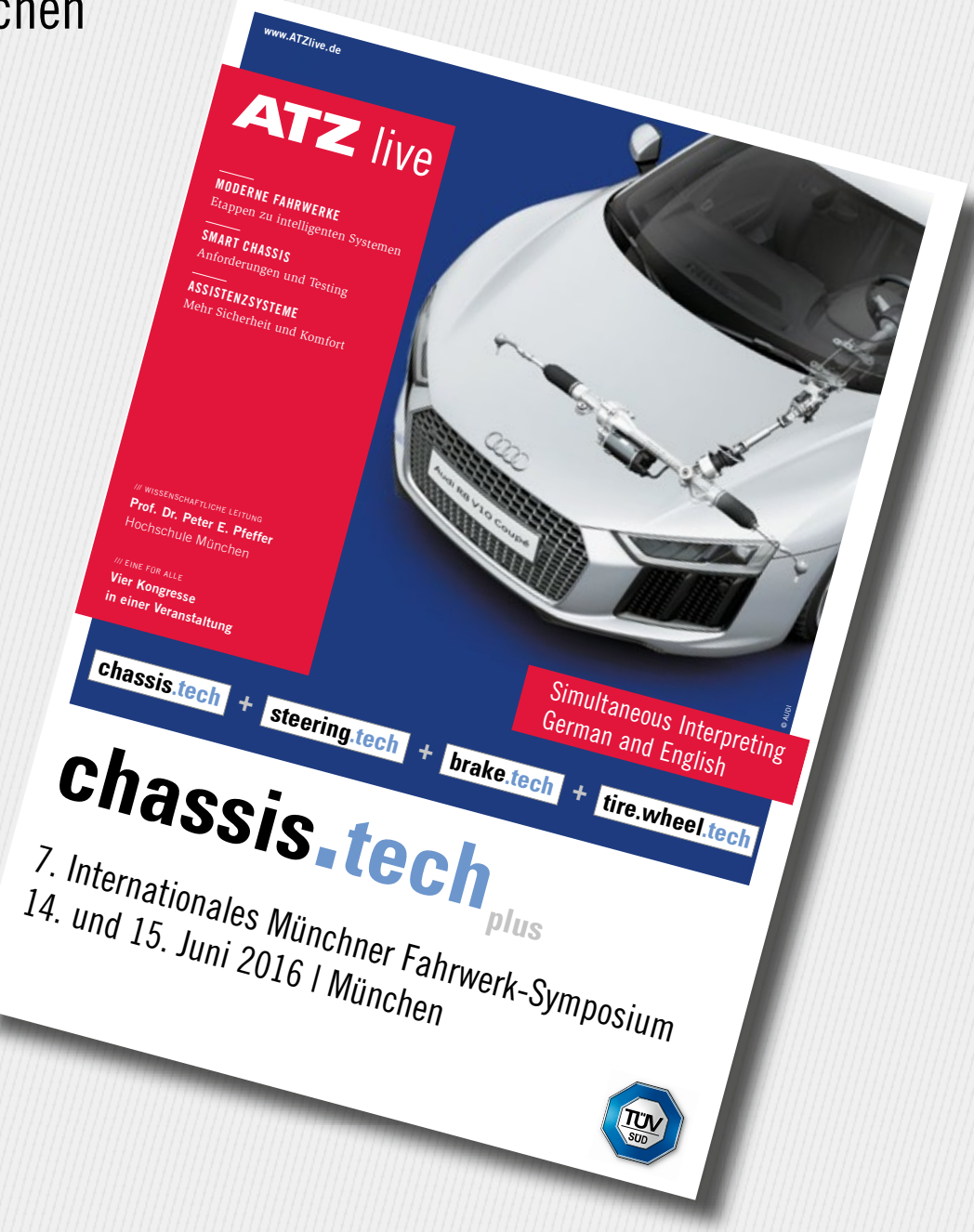


